\title{
Comparison of Secondary, Backscattered and Low Loss Electron Imaging for Dimensional Measurements in the Scanning Electron Microscope - Part $2^{[1,2]}$
}

\author{
Michael T. Postek ${ }^{1}$, John Villarrubia ${ }^{2}$, András E. Vladár ${ }^{3}$ and Atsushi Muto ${ }^{4}$ \\ ${ }^{1-3 .}$ National Institute of Standards and Technology, Physical Measurement Laboratory, Gaithersburg, \\ MD \\ ${ }^{4}$ Hitachi High Technologies America, Clarksburg, MD
}

In many research and production environments, a great deal of dimensional metrology, characterization and process control is accomplished using scanning electron microscopes (SEM). The accuracy of these SEM measurements has always been important, but is often overshadowed by two other main measurement drivers: throughput and precision. It is slow and often tedious to achieve accuracy and, so it is often ignored, especially in the production environment. Accuracy of a measurement is becoming more of an abiding concern as sub-10 nm semiconductor structures are routinely produced. Hence, the metrology error budget has shrunk, and has become atomic scale. Clever new measurement and signal collection methods applied to sub-10 nm metrology must be sought for all types of semiconductor nanostructures, nanomaterials and nano-enabled materials to ultimately achieve the needed accurate measurements.

The quality of the SEM metrology depends on a number of interrelated factors such as: the acquired image and how it is influenced by vibration, drifts, sample contamination and charging, etc. In addition, accounting for specimen-electron beam interactions and their contributions to the imaging must also be considered. New acquisition methods and successful mitigation of the previously mentioned detrimental effects can alleviate some of the imaging problems. But, another key element is the application of advanced electron beam-solid state interaction modeling, such as the NIST JMONSEL [3] model to interpret and account for the physics of the signal generation, and help to understand and minimize the various contributions to measurement inaccuracy.

The first part of this work involved a fundamental comparison of secondary (SE), backscattered (BSE) and low-loss (LLE) electron signals acquired on a new instrument that has high-angle BSE and energyfiltered LLE detectors. Early work indicated that the LLE signal could be advantageous for metrology [4]. When that work was first done, it was very difficult to obtain the needed information because of poor signal-to-noise ratio (SNR) and other instrument-specific geometric limitations. LLE imaging is difficult because it involves the collection of energy filtered BSE that have undergone only minimal inelastic interactions with a sample and therefore carry high-resolution, surface-specific information [58]. Early work, using the conventional backscattered electron signal was shown to be beneficial at low landing energies using a microchannel-plate electron detector [9]. In that work, collection and comparison of BSE and SE images of line structures demonstrated that the former measured about $10 \%$ narrower [9]. Due to the enhanced emission of low-energy (typically less than $10 \mathrm{eV}$ ) electrons at the sides and corners, there are common circumstances in which the SE intensity increases more abruptly at an edge than the BSE intensity. If width assignments are based on an intensity threshold, as is often the case, SE images would then be interpreted as showing a wider feature than the BSE image. It was anticipated that the LLE signal would provide results similar to the BSE results. A Hitachi SU 8230 [2] FESEM, equipped with a high-angle and energy-filtered backscattered electron detector, was used to compare the SE, BSE and LLE signals for dimensional measurements of the NIST RM 8820 
magnification calibration sample [10]. The design of the field emission SEM incorporating a new in-lens energy filtered detector improves the LLE signal-to-noise ratio and reduces the geometrical limitations of the early LLE detectors.

In this second part of the work, we continued the analysis of the dimensional measurements. Simulations with JMONSEL, an electron microscope simulator, indicate that the nanometer-scale differences observed on this sample can be explained by the different convolution effects of a beam with finite size on signals with different symmetry (the SE signal's characteristic peak vs. the BSE or LLE signal's characteristic step). This effect is too small to explain the $>100 \mathrm{~nm}$ discrepancies that had been observed in earlier work on different samples. However, additional modeling indicates that those discrepancies can be explained by considering the much larger sidewall angles of the earlier samples, coupled with the different response of SE vs. BSE/LLE profiles to such wall angles. Clearly, serious measurement errors would be encountered in such cases if measurement algorithms were applied blindly without considering the underlying physics provided by applying model-based metrology.

References:

[1]Contribution of the National Institute of Standards and Technology; not subject to copyright. [2]Certain commercial equipment is identified in this report to adequately describe the experimental procedure. Such identification does not imply recommendation or endorsement by the National Institute of Standards and Technology, nor does it imply that the equipment identified is necessarily the best available for the purpose.

[3] J. S. Villarrubia et al., Ultramicroscopy 154 (2015) p. 15.

[4] M. T. Postek et al., SCANNING 23(5), (2001), p. 298.

[5] O. C. Wells, Appl Phys Lett 16(4), (1970), p.151.

[6] O. C. Wells, Appl Phys Lett 19(7), (1971), p. 232.

[7] O. C. Wells, Scan Electron Microsc, 1, IITRI Chicago, (1972), p. 43.

[8] O. C. Wells, Appl. Phys. Lett. 49(13), (1986) p. 764.

[9] M. T. Postek et al., Rev Sci. Instrum, 61(12), (1990), p. 3750.

[10]https://www-s.nist.gov/srmors/view_detail.cfm?srm $=8820$
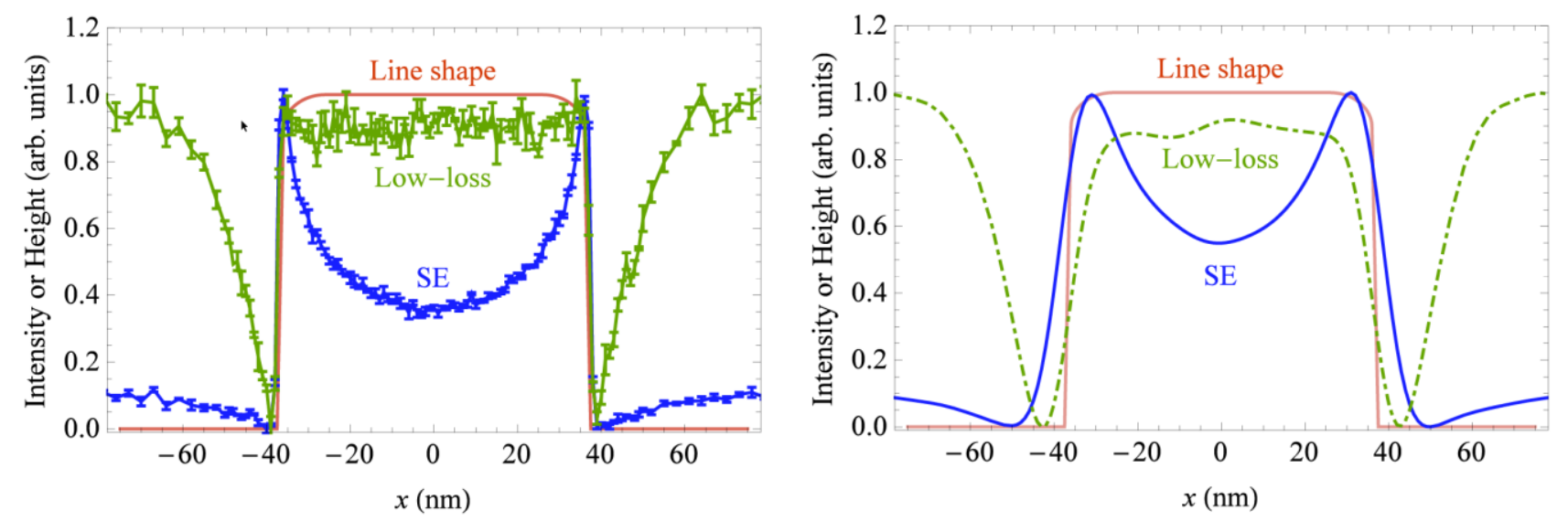

Figure 1. JMONSEL modeled data. (left) Modeling of an ideal silicon structure with vertical walls mimicking the structure of RM 8820 with "ideal" instrument conditions. (right) Modeling of the same structure, but with "real-world" electron beam conditions and sample conditions included. 